\title{
Inclusion of Angular Momentum During Planning for Capture Point Based Walking*
}

\author{
Tim Seyde ${ }^{1}$, Apoorv Shrivastava ${ }^{1}$, Johannes Englsberger ${ }^{2}$, Sylvain Bertrand ${ }^{1}$, Jerry Pratt $^{1}$, Robert J. Griffin ${ }^{1}$
}

\begin{abstract}
When walking at high speeds, the swing legs of robots produce a non-negligible angular momentum rate. To accommodate this, we provide a reference trajectory generator for bipedal walking that incorporates predicted centroidal angular momentum at the planning stage. This can be done efficiently as the Centroidal Moment Pivot (CMP), Instantaneous Capture Point (ICP) and the center of mass (CoM) all have closedform trajectory solutions due to their linear dynamics. This is then used to produce smooth, continuous trajectories. We furthermore provide a lightweight model to estimate angular momentum as induced during leg swing of the gait cycle. Our proposed trajectory generator is tested thoroughly in simulation and has been shown to successfully operate on the real hardware.
\end{abstract}

\section{INTRODUCTION}

Bipedal locomotion is a challenging problem: a highdimensional system needs to make and break friction-limited unilateral ground contacts in order to control underactuated, nonlinear dynamics that exist in hybrid form. While the underlying problem features are highly complex, the main objective can be condensed to making the center of mass (CoM) track a desired path along which footsteps are placed for support. The Linear Inverted Pendulum (LIP) model put forward by Kajita et al. [1] follows this idea by projecting the general system dynamics to the CoM. In combination with the concept of the Zero Moment Point (ZMP) from Vukobratovic et al. [2], locomotion planners result that show sufficient performance under runtime constraints (e.g. [3]).

The introduction of the Instantaneous Capture Point (ICP) put forward by Pratt et al. [4] offered a concise formulation of footstep placement for recovery from external perturbations. Its usage in locomotion planning has seen widespread application in the planar case (e.g. [5] and [6]) and through its generalization to the Divergent Component of Motion (DCM) also the 3D case (e.g. [7] and [8]). The resulting reference trajectories generated by these planners are then tracked using feedback controllers that can leverage knowledge of the actual system dynamics. By employing solely the LIP in the planning stage, these approaches introduce the assumption of the center of pressure $(\mathrm{CoP})$ coinciding with the Centroidal Moment Pivot (CMP) and therefore result in

\footnotetext{
*This work was funded through the National Robotics Initiative by the National Aeronautics and Space Administration (grant number NNX12AP97G)

${ }^{1}$ The author is with the Florida Institute for Human and Machine Cognition (IHMC), 40 S Alcaniz St, Pensacola, FL 32502, United States

${ }^{2}$ The author is with the Institute of Robotics and Mechatronics, German Aerospace Center (DLR), 82234 Wessling, Germany

Email: $\{$ tseyde, ashrivastava, sbertrand, jpratt, rgriffin\}@ihmc.us, johannes.englsbergerddlr.de
}
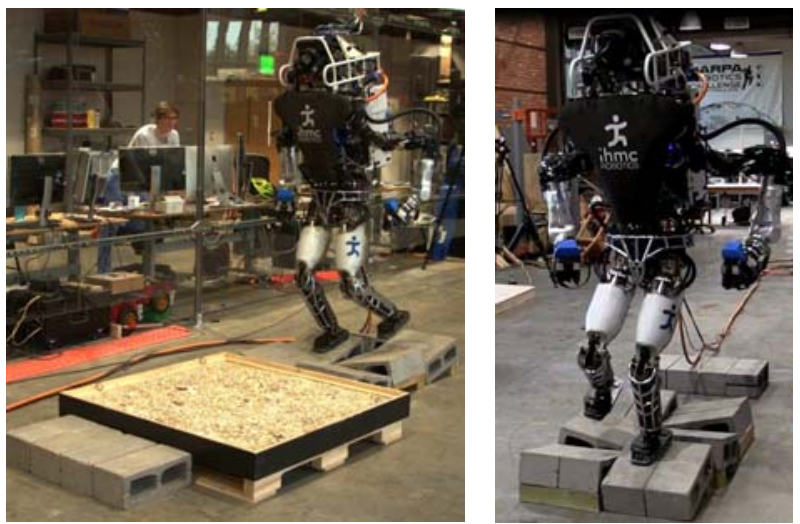

Fig. 1: Atlas walking over rough terrain. When considering very dynamic motions, including angular momentum at the planning stage can be advantageous.

minimization of change in centroidal angular momentum. This can be at odds with the desired swing leg motion and unnecessarily constrains the CMP to the support polygon.

The effect of centroidal angular momentum during the human gait cycle has been studied in [9]. However, there have only been few attempts to explicitly account for its effects during the planning stage of bipedal locomotion. Pratt et al. [4] and Lee et al. [10] discuss extensions to the LIP that try to model centroidal momenta. Dai et al. [11] and Herzog et al. [12] put forward methods to include approximations of the angular momentum to allow for more consistent motion planning. When aiming at generating fast and dynamic locomotion for robots with non-negligible mass distribution in the extremities, the explicit inclusion of these effects becomes inevitable for improving performance. In case of bipedal walking, the CMP can offer a straight forward entry point to include angular momentum during planning.

In this work, we provide an extension to the smooth ICP reference generator provided by Englsberger et al. [13] that makes it easy to include angular momentum references at the planning stage. We furthermore provide an inexpensive scheme for generating an approximation of the centroidal torque induced by swing leg motion. A short overview over the relevant theory is provided in Section II. Section III then introduces our formulation of the CMP, ICP and the CoM reference trajectory generators, and shows how we handle reference adjustment for replanning. Simulation results are provided in Section IV, while real hardware application is discussed in Section V. Section VI concludes the paper and provides a short description of possible future work. 


\section{RELEVANT THEORY AND PREVIOUS WORK}

The underlying planning algorithm was presented in [14] based on the the DCM. Under the assumption of constant CoM height during planning, the DCM is equivalent to the ICP. In the following, we provide an overview of the relevant theoretical background.

\section{A. LIP dynamics and the ICP}

The ICP is a transformation of the CoM state defined as

$$
\boldsymbol{\xi}=\boldsymbol{x}+\frac{1}{\omega_{0}} \dot{\boldsymbol{x}}
$$

where $\boldsymbol{\xi}=\left[\xi_{x}, \xi_{y}\right]^{T}$ is the ICP position, $\boldsymbol{x}=[x, y]^{T}$ is the CoM position, and $\omega_{0}=\sqrt{g / \Delta z_{C o M}}$ is the pendulum constant for the LIP. Reordering (1), we find the CoM dynamics as

$$
\dot{\boldsymbol{x}}=\omega_{0}(\boldsymbol{\xi}-\boldsymbol{x}) .
$$

The CoM therefore has stable first order dynamics and converges towards the ICP. Differentiating (1) and using Newton's $2^{\text {nd }}$ law, we find the ICP dynamics as

$$
\dot{\boldsymbol{\xi}}=\omega_{0}\left(\boldsymbol{\xi}-\boldsymbol{r}_{\mathrm{CMP}}\right) \text {. }
$$

The CMP encodes the total linear force acting on the CoM

$$
\boldsymbol{F}_{C o M}=m \omega_{0}^{2}\left(\boldsymbol{x}-\boldsymbol{r}_{\mathrm{CMP}}\right) \text {. }
$$

Considering (3), the ICP displays unstable first order dynamics and diverges from the CMP. An ICP tracking control law can then be formulated to yield closed-loop stable ICP dynamics together with inherently stable CoM dynamics.

In an effort to guarantee feasibility of the CoP trajectories, Englsberger et al. presented a smooth DCM and VRP reference trajectory generator in [13]. A smooth VRP reference is achieved by using spline interpolation between VRP waypoints, and its corresponding DCM reference through integration. During the planning stage, ZMP and CMP references coincide based on the assumption of negligible angular momentum.

\section{B. Momentum-based control}

The underlying momentum-based control framework is described in detail in [15]. A quadratic program (QP) is used to find optimized joint accelerations and ground reaction wrenches based on the desired motion tasks, ground contact information and a centroidal momentum objective. During walking, the QP tries to achieve the desired control CMP through linear momentum rate of change and to follow the reference swing leg motion. Conventional approaches based on LIP dynamics assume a massless swing leg during planning and therefore choose the reference CMP to coincide with the reference CoP. When applied to a multi-body robot with distributed masses, this can lead to degraded performance.

In the following, we therefore introduce a reformulation of the waypoint-centric approach provided in [13] to a polynomial-centric approach that explicitly incorporates angular momentum references, together with a light-weight approximation of angular momentum initialized by a three mass model simplification of the overall robot model.
III. SMOOTH ICP REFERENCES GENERATION CONSISTENT WITH ANGULAR MOMENTUM OBJECTIVES

This section provides the formulation of the CMP, ICP and CoM reference trajectory generators. To avoid clutter, we make use of the decoupled nature of the LIP dynamics and write corresponding equations in their scalar form. They can then be applied in both $x$ and $y$ direction. We furthermore show how to adjust the CoP reference to account for footstep replanning or tracking error.

\section{A. CMP reference generation}

As shown in [16], the CMP can be expressed as the sum of the ZMP and the moment about the CoM

$$
\begin{aligned}
& r_{\mathrm{CMP}, x}=r_{\mathrm{ZMP}, x}+\frac{\tau_{y}\left(\boldsymbol{r}_{\mathrm{CoM}}\right)}{F_{G R, z}} \\
& r_{\mathrm{CMP}, y}=r_{\mathrm{ZMP}, y}-\frac{\tau_{x}\left(\boldsymbol{r}_{\mathrm{CoM}}\right)}{F_{G R, z}},
\end{aligned}
$$

where $\boldsymbol{\tau}\left(\boldsymbol{r}_{\mathrm{CoM}}\right)$ is the CoM torque and $\boldsymbol{F}_{G R}$ the ground reaction force. Assuming flat ground walking, the $\mathrm{CoP}$ coincides with the ZMP and will be used in its place. The CoP and angular momentum reference can then be considered separately in the CMP generation. Following the smooth trajectory generation put forward in [13], we express both terms using splines. The combination of the two separately generated references is then a simple addition of the coefficients. We formulate the CMP reference trajectories as a series of $n_{\phi}$ smooth splines over the preview horizon. Each spline $r_{\mathrm{CMP}, r e f, \phi}$ is of order $n_{\mathrm{CMP}}$ and can be represented as

$$
\begin{aligned}
r_{\mathrm{CMP}, \text { ref }, \phi}\left(t_{\phi}\right) & =\sum_{j=0}^{n_{\tau}} a_{j} t_{\phi}^{j}+\sum_{j=0}^{n_{\mathrm{CoP}}} b_{j} t_{\phi}^{j}=\sum_{j=0}^{n_{\mathrm{CMP}}} p_{j} t_{\phi}^{j} \\
& =\boldsymbol{t}^{T}\left(t_{\phi}\right) \cdot \boldsymbol{p}_{\phi},
\end{aligned}
$$

where $t_{\phi}$ is the local time on segment $\phi, t\left(t_{\phi}\right)$ is its vectorized form and $\boldsymbol{p}_{\phi}$ is the vector of polynomial coefficients. The latter consists of the individual CMP coefficients $p_{j}$ expressed as a linear combination of the torque coefficients $a_{j}$ and the CoP coefficients $b_{j}$ on segment $\phi$, such that $n_{\mathrm{CMP}}=\max \left\{n_{\tau}, n_{\mathrm{CoP}}\right\}$. Separating the constant coefficients vector from the time-varying vector in this form will be advantageous for the ICP reference generation. For readability, we will drop the segment index $\phi$ from the polynomial coefficient vector and simply write $\boldsymbol{p}$.

1) CoP trajectory: The $\mathrm{CoP}$ reference trajectories are generated through spline interpolation between waypoints. Similar to [17], CoP waypoints are placed in the heel and toe of each footstep. To allow for a more nuanced control of the reference, additional waypoints are placed in the ball of the foot and at the centroid of the double support polygon. Letting $l \in\{$ heel,ball,toe 1, toe 2 ,cent $\}$ denote the waypoint type, we define the respective locations for footstep $i$ as

$$
\boldsymbol{r}_{\mathrm{CoP}, i, l}=\boldsymbol{c}_{i}+K_{l}^{1}\left(\boldsymbol{c}_{i+1}-\boldsymbol{c}_{i}\right)+K_{l}^{-1}\left(\boldsymbol{c}_{i}-\boldsymbol{c}_{i-1}\right)+\boldsymbol{\delta}_{i, l},
$$

where $c_{i}$ is the centroid of the $i$-th footstep, $K_{l}^{1}$ and $K_{l}^{-1}$ are step length offset factors for the next and previous step 

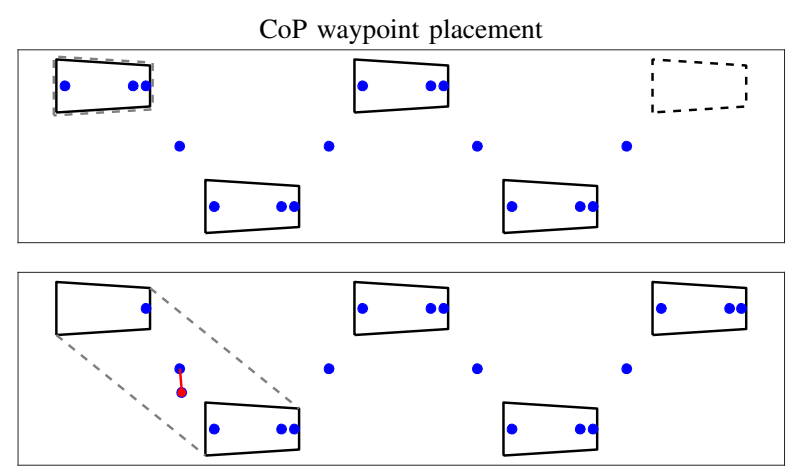

Fig. 2: Footstep plan with reference CoP waypoints (blue) and support polygon (dotted grey). Top: single support (SS). Bottom: double support (DS) with adjusted CoP waypoint (red).

length respectively, and $\boldsymbol{\delta}_{i, l}$ is a tunable offset to alter the gait dynamics. Ball and toe $\mathrm{CoP}$ waypoints are associated with the single support (SS) phase, whereas heel and centroid $\mathrm{CoP}$ waypoints are associated with the double support (DS) phase. Every swing and transfer phase is therefore split into at least 2 segments, a structure that will be exploited in Section III-D to ensure continuity between successive plans. The planned time duration for moving from one waypoint to the next waypoint is a parameter tuned as a percentage of the swing or transfer durations. The trajectory segment connecting consecutive waypoints is generated using spline interpolation, as in [13], based on the formula

$$
\boldsymbol{r}_{\mathrm{CoP}, \phi}\left(t_{\phi}\right)=\left(1-f_{\phi}\left(t_{\phi}\right)\right) \boldsymbol{r}_{\mathrm{CoP}, \phi, 0}+f_{\phi}\left(t_{\phi}\right) \boldsymbol{r}_{\mathrm{CoP}, \phi, T}
$$

where $\boldsymbol{r}_{\mathrm{CoP}, \phi, 0}=\boldsymbol{r}_{\mathrm{CoP}, \phi}\left(t_{\phi, 0}\right)$ is the segment's initial $\mathrm{CoP}$, $\boldsymbol{r}_{\mathrm{CoP}, \phi, T}=\boldsymbol{r}_{\mathrm{CoP}, \phi}\left(T_{\phi}\right)$ is the segment's final CoP, and $f_{\phi}\left(t_{\phi}\right)$ is a function of pre-defined order that continuously interpolates from 0 to 1 with appropriate terminal derivatives set to zero. Previous approaches often employed a constant or linear reference $\mathrm{CoP}$ during planning. Here, we use a linear interpolating function to ensure reference continuity, while retaining comparability with previous implementations.

2) Angular momentum: In order to estimate the rate of change of angular momentum, the robot is approximated using the three mass model taken from Takenaka et al. [18]. Here, the robot is represented via lumped masses at the main body $m_{\text {body }}$, the swing leg $m_{\text {swing }}$ and the stance leg $m_{\text {stance }}$ (Fig. 3). A prediction of the centroidal angular momentum can then be generated based on estimations of the lumped masses' trajectories. Here, we model these trajectories using quintic splines under boundary constraints. For the main body on segment $i$, we impose the boundary conditions

$$
\begin{array}{ll}
\hat{\boldsymbol{x}}_{\text {body }, i}\left(t_{i, 0}\right)=\boldsymbol{r}_{\mathrm{CoP}, i, c e n t} & \hat{\boldsymbol{x}}_{\text {body }, i}\left(T_{i}\right)=\boldsymbol{r}_{\mathrm{CoP}, i+1, \text { cent }}, \\
\dot{\hat{\boldsymbol{x}}}_{\text {body }, i}\left(t_{i, 0}\right)=\dot{\hat{\boldsymbol{r}}}_{\mathrm{CoP}, i, i, c e n t} & \dot{\hat{\boldsymbol{x}}}_{\text {body }, i}\left(T_{i}\right)=\dot{\hat{\boldsymbol{r}}}_{\mathrm{CoP}, i+1, c e n t}, \\
\ddot{\tilde{\boldsymbol{x}}}_{\text {body }, i}\left(t_{i, 0}\right)=\mathbf{0} & \ddot{\hat{\boldsymbol{x}}}_{\text {body }, i}\left(T_{i}\right)=\mathbf{0},
\end{array}
$$

where $\dot{\boldsymbol{r}}_{\mathrm{CoP}, j, \text { cent }}=\frac{r_{\mathrm{CoP}, j+1, \text { heel }}-\boldsymbol{r}_{\mathrm{COP}, j, t o e 2}}{t_{j+1, \text { heel }, 0}-t_{j, \text { toe }, 0}}$ denotes the average velocity of shifting the CoP from one foot to the next. For the

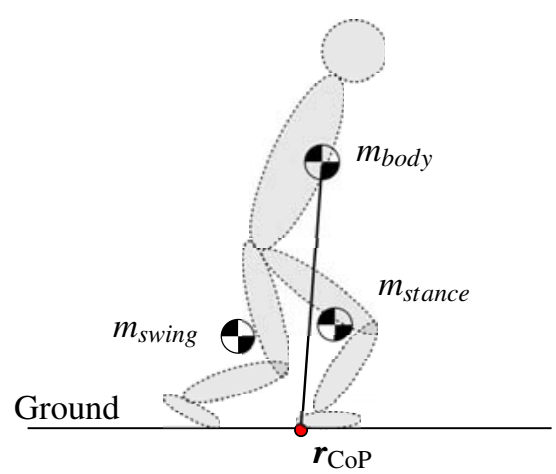

Fig. 3: Three mass model with lumped masses for the main body, $m_{\text {body }}$, the stance leg, $m_{\text {stance }}$, and the swing leg $m_{\text {stance }}$.

swing and the stance leg we impose the boundary conditions

$$
\begin{array}{ll}
\hat{\boldsymbol{x}}_{\text {swing }, i}\left(t_{i, 0}\right)=\boldsymbol{c}_{i} & \hat{\boldsymbol{x}}_{\text {swing }, i}\left(T_{i}\right)=\boldsymbol{c}_{i+2}, \\
\hat{\boldsymbol{x}}_{\text {swing }, i}^{(n)}\left(t_{i, 0}\right)=\mathbf{0} & \hat{\boldsymbol{x}}_{\text {swing }, i}^{(n)}\left(T_{i}\right)=\mathbf{0}, \\
\hat{\boldsymbol{x}}_{\text {stance }, i}\left(t_{i, 0}\right)=\boldsymbol{c}_{i+1} & \hat{\boldsymbol{x}}_{\text {stance }, i}\left(T_{i}\right)=\boldsymbol{c}_{i+1}, \\
\hat{\boldsymbol{x}}_{\text {stance }, i}^{(n)}\left(t_{i, 0}\right)=\mathbf{0} & \hat{\boldsymbol{x}}_{\text {stance }, i}^{(n)}\left(T_{i}\right)=\mathbf{0},
\end{array}
$$

with $n \in\{1,2\}$. For the vertical component of the swing leg motion, a quadratic polynomial parameterized using the maximum height above the ground, $z_{\max }$, is used

$$
\hat{\boldsymbol{x}}_{z, \text { swing }, i}(t)=4 \tau(1-\tau) z_{\max } .
$$

Here, $\tau=\frac{t}{T_{\text {swing }}}$ denotes the normalized swing time. The resultant lumped mass trajectories are then used to compute the estimated centroidal angular momentum trajectory from

$$
\boldsymbol{L}=\sum_{\text {foot }} m_{\text {foot }}\left(\hat{\boldsymbol{x}}_{\text {body }}-\hat{\boldsymbol{x}}_{\text {foot }}\right) \times\left(\dot{\hat{\boldsymbol{x}}}_{\text {body }}-\dot{\hat{\boldsymbol{x}}}_{\text {foot }}\right),
$$

where foot $\in\{$ swing, stance $\}$, and $\hat{x}_{\text {body }}$ and $\hat{x}_{\text {foot }}$ denote the segmented trajectories. The lumped masses can then be tuned based on the real system to minimize disagreement between (9) and (15). A fast Fourier transform is used to speed up the calculation of the polynomial cross product. The angular momentum contribution to the CMP in (6) can then be computed by differentiating the segmented trajectory resulting from (12) and using it in (5).

\section{B. ICP reference generation}

Using the resulting CMP reference trajectories from the previous section, we can integrate the unstable ICP dynamics from (3) backwards in time. The resulting ICP reference trajectory formulation on segment $\phi$ is provided as

$$
\begin{aligned}
\xi_{\text {ref }, \boldsymbol{\phi}}\left(t_{\phi}\right)= & \sum_{j=0}^{n} \omega_{0}^{-j}\left(\boldsymbol{t}^{(j)^{T}}\left(t_{\phi}\right)-\boldsymbol{t}^{(j)^{T}}\left(T_{\phi}\right) e^{\omega_{0}\left(t_{\phi}-T_{\phi}\right)}\right) \cdot \boldsymbol{p} \\
& +e^{\omega_{0}\left(t_{\phi}-T_{\phi}\right)} \xi_{\text {ref }, \phi}\left(T_{\phi}\right),
\end{aligned}
$$

where $\boldsymbol{t}^{(j)}$ is the $j^{\text {th }}$ time vector derivative and $T_{\phi}$ the final time. Analogously to [13], we group the time-dependent 
terms as vectors $\boldsymbol{\alpha}_{\phi}\left(t_{\phi}\right), \boldsymbol{\beta}_{\phi}\left(t_{\phi}\right)$ and scalar $\gamma_{\phi}\left(t_{\phi}\right)$

$$
\begin{aligned}
\xi_{r e f, \phi}\left(t_{\phi}\right) & =\left(\boldsymbol{\alpha}_{\phi}\left(t_{\phi}\right)-\boldsymbol{\beta}_{\phi}\left(t_{\phi}\right)\right) \cdot \boldsymbol{p}+\gamma_{\phi}\left(t_{\phi}\right) \xi_{r e f, \phi}\left(T_{\phi}\right), \\
\boldsymbol{\alpha}_{\phi}\left(t_{\phi}\right) & =\sum_{j=0}^{n} \omega_{0}^{-j} \boldsymbol{t}^{(j)^{T}}\left(t_{\phi}\right) \\
\boldsymbol{\beta}_{\phi}\left(t_{\phi}\right) & =\sum_{j=0}^{n} \omega_{0}^{-j} \boldsymbol{t}^{(j)^{T}}\left(T_{\phi}\right) e^{\omega_{0}\left(t_{\phi}-T_{\phi}\right)} \\
\gamma_{\phi}\left(t_{\phi}\right) & =e^{\omega_{0}\left(t_{\phi}-T_{\phi}\right)}
\end{aligned}
$$

\section{CoM reference generation}

Based on the ICP reference trajectory derived in the previous section, we can now integrate the stable CoM dynamics from (2) forward in time to find a closed form solution provided by

$$
\begin{aligned}
& x_{r e f, \phi}\left(t_{\phi}\right)=\left(\boldsymbol{\alpha}_{\mathrm{CoM}, \phi}\left(t_{\phi}\right)-\boldsymbol{\beta}_{\mathrm{CoM}, \phi}\left(t_{\phi}\right)\right) \cdot \boldsymbol{p} \\
&+\delta_{\mathrm{CoM}, \phi}\left(t_{\phi}\right)\left(\xi_{r e f, \phi}\left(T_{\phi}\right)-\boldsymbol{\alpha}_{\phi}\left(T_{\phi}\right) \cdot \boldsymbol{p}\right) \\
&+\gamma_{\mathrm{CoM}, \phi}\left(t_{\phi}\right) x_{r e f, \phi}\left(t_{\phi, 0}\right), \\
& \boldsymbol{\alpha}_{\mathrm{CoM}, \phi}\left(t_{\phi}\right)=\sum_{i=0}^{n} \sum_{j=i}^{n}(-1)^{i} \omega_{0}^{-j} \boldsymbol{t}^{(j)^{T}}\left(t_{\phi}\right), \\
& \boldsymbol{\beta}_{\mathrm{CoM}, \phi}\left(t_{\phi}\right)=\sum_{i=0}^{n} \sum_{j=i}^{n}(-1)^{i} \omega_{0}^{-j} \boldsymbol{t}^{(j)^{T}}\left(t_{\phi, 0}\right) e^{\omega_{0}\left(t_{\phi, 0}-t_{\phi}\right)}, \\
& \gamma_{\mathrm{CoM}, \phi}\left(t_{\phi}\right)=e^{\omega_{0}\left(t_{\phi, 0}-t_{\phi}\right)}, \\
& \delta_{\mathrm{CoM}, \phi}\left(t_{\phi}\right)=e^{\omega_{0}\left(t_{\phi, 0}-T_{\phi}\right)} \sinh \left(\omega_{0}\left(t_{\phi}-t_{\phi, 0}\right)\right),
\end{aligned}
$$

where $t_{\phi, 0}$ is the segment's initial time. This analytic formulation can then be used to gain better insights into the intended motion and to preview possible CoM constraint violations.

\section{Reference adjustment for continuity}

Updating the footstep plan re-initializes the ICP backward iteration algorithm. Without actively enforcing boundary constraints on smoothness, consecutive updates to the plan can result in discontinuous references. For example, early swing termination due to premature foot touchdown will leave a gap between the achieved final swing reference ICP and the following initial transfer reference ICP. In order to guarantee smoothness of the ICP reference, we recompute the CMP reference to offset this initial discontinuity. This is achieved by shifting the $\mathrm{CoP}$ waypoint at the end of the current transfer phase's first segment as shown in Fig. 2 (bottom), thus generating new polynomial coefficient vectors on the first and second transfer segment. We enforce the following smoothness constraints up to the $k^{\text {th }}$ derivative, where $k=\left\lfloor n_{\mathrm{CMP}} / 2\right\rfloor$ :

- C1: ICP transition between first transfer segment and previous final swing segment, $\boldsymbol{\xi}_{0,0}=\boldsymbol{\xi}_{-1, T}$.

- C2: CoP transition between first transfer segment and previous final swing segment, $\boldsymbol{r}_{\mathrm{CoP}, 0,0}=\boldsymbol{r}_{\mathrm{CoP},-1, T}$.

- C3: CoP transition between first transfer segment and second transfer segment, $\boldsymbol{r}_{\mathrm{CoP}, 1,0}=\boldsymbol{r}_{\mathrm{CoP}, 0, T}$.

- C4: CoP transition out of the second transfer segment, $\boldsymbol{r}_{\mathrm{CoP}, 2,0}=\boldsymbol{r}_{\mathrm{CoP}, 1, T}$.
Here we use the notation $\boldsymbol{\xi}_{\phi, t_{\phi}}$ with $\phi=0$ referring to the first (initial) transfer segment. When starting from stance, conditions $\mathrm{C} 1$ and $\mathrm{C} 2$ are both set to the corresponding current $\mathrm{CoP}$ values. Due to condition $\mathrm{C} 4$, the adjustment will only affect the first two transfer segments of the current plan and the remaining segments will be left untouched. The linear system of equations to be solved for ensuring smoothness of the $j^{\text {th }}$ derivative is given by

$$
\left[\begin{array}{c}
\xi_{-1, T}^{(j)}-\gamma_{0,0}^{(j)} \gamma_{1,0} \xi_{1, T} \\
r_{\mathrm{CoP}, 0,0}^{(j)} \\
0 \\
r_{\mathrm{CoP}, 1, T}^{(j)}
\end{array}\right]=\left[\begin{array}{cc}
\boldsymbol{\Delta}_{0,0}^{(j)} & \boldsymbol{\gamma}_{0,0}^{(j)}\left(\boldsymbol{\Delta}_{1,0}\right) \\
\boldsymbol{t}_{0,0}^{(j)^{T}} & \mathbf{0} \\
-\boldsymbol{t}_{0, T}^{(j)^{T}} & \boldsymbol{t}_{1,0}^{(j)^{T}} \\
\mathbf{0} & \boldsymbol{t}_{1, T}^{(j)^{T}}
\end{array}\right]\left[\begin{array}{c}
\tilde{\boldsymbol{p}}_{0} \\
\tilde{\boldsymbol{p}}_{1}
\end{array}\right]
$$

where $\boldsymbol{\Delta}_{\phi, t_{\phi}}=\boldsymbol{\alpha}_{\phi, t_{\phi}}-\boldsymbol{\beta}_{\phi, t_{\phi}}$. Solving for the adjusted polynomial coefficient vectors of the first two segment, $\tilde{\boldsymbol{p}}_{0}$ and $\tilde{\boldsymbol{p}}_{1}$, we can define the general adjusted polynomial coefficient vector for each segment as

$$
\boldsymbol{p}_{a d j, \phi}= \begin{cases}\tilde{\boldsymbol{p}}_{\phi}, & \phi \in\{0,1\} \\ \boldsymbol{p}_{\phi}, & \text { otherwise }\end{cases}
$$

Using these adjusted vectors and leveraging linearity in the coefficients, the adjusted ICP trajectory takes the form

$$
\boldsymbol{\xi}_{\text {ref,adj,t }}=\boldsymbol{\Delta}_{t} \cdot\left(\boldsymbol{p}_{\text {adj } j}+\boldsymbol{a}\right)+\gamma_{t} \boldsymbol{\xi}_{\text {ref,adj }, T},
$$

where we dropped the explicit dependence on $\phi$ to avoid clutter and $\boldsymbol{a}$ is the stacked polynomial coefficient vector of the torque reference, as introduced in Section III-A.

\section{E. ICP control}

The desired control law is then formulated on the CMP level, similar to [13]. It consists of a feed-forward term using the reference CMP and a feed-back term accounting for reference ICP tracking errors. To account for replanning, the adjusted references introduced in the previous section are employed. The control CMP is given by

$$
\boldsymbol{r}_{\mathrm{CMP}}=\boldsymbol{r}_{\mathrm{CMP}, a d j, \phi}+\left(\boldsymbol{I}+\boldsymbol{K}_{\xi} \omega_{0}^{-1}\right)\left(\boldsymbol{\xi}-\boldsymbol{\xi}_{a d j, \phi}\right),
$$

where $\boldsymbol{K}_{\boldsymbol{\xi}}$ is a positive definite gain matrix. To avoid unreasonably large control inputs, the control CMP is limited to lie within an enlargement of the support polygon grown $0.06 \mathrm{~m}$ in every direction. This value offered a good trade-off between allowing sufficient angular momentum and rejecting noisy sensor data.

\section{Simulation RESUlts}

\section{A. Ideal Planning}

In the following we compare the proposed planner to the continuous double support (CDS) planner, the continuous heel-to-toe shift (HT) planner (both [17]) and the smooth ICP planner put forward in [13]. Based on the ideal LIP equation, we generate ICP reference trajectories for a 5-step straight walking plan. The step plan parameters are $m=150 \mathrm{~kg}, t_{S S}=$ $0.6 \mathrm{~s}, t_{D S}=0.2 \mathrm{~s}, l_{\text {step }}=0.5 \mathrm{~m}$ and $w_{\text {step }}=0.25 \mathrm{~m}$, while the initial and final transfer times are both 1s. Based on Fig. 4, it 

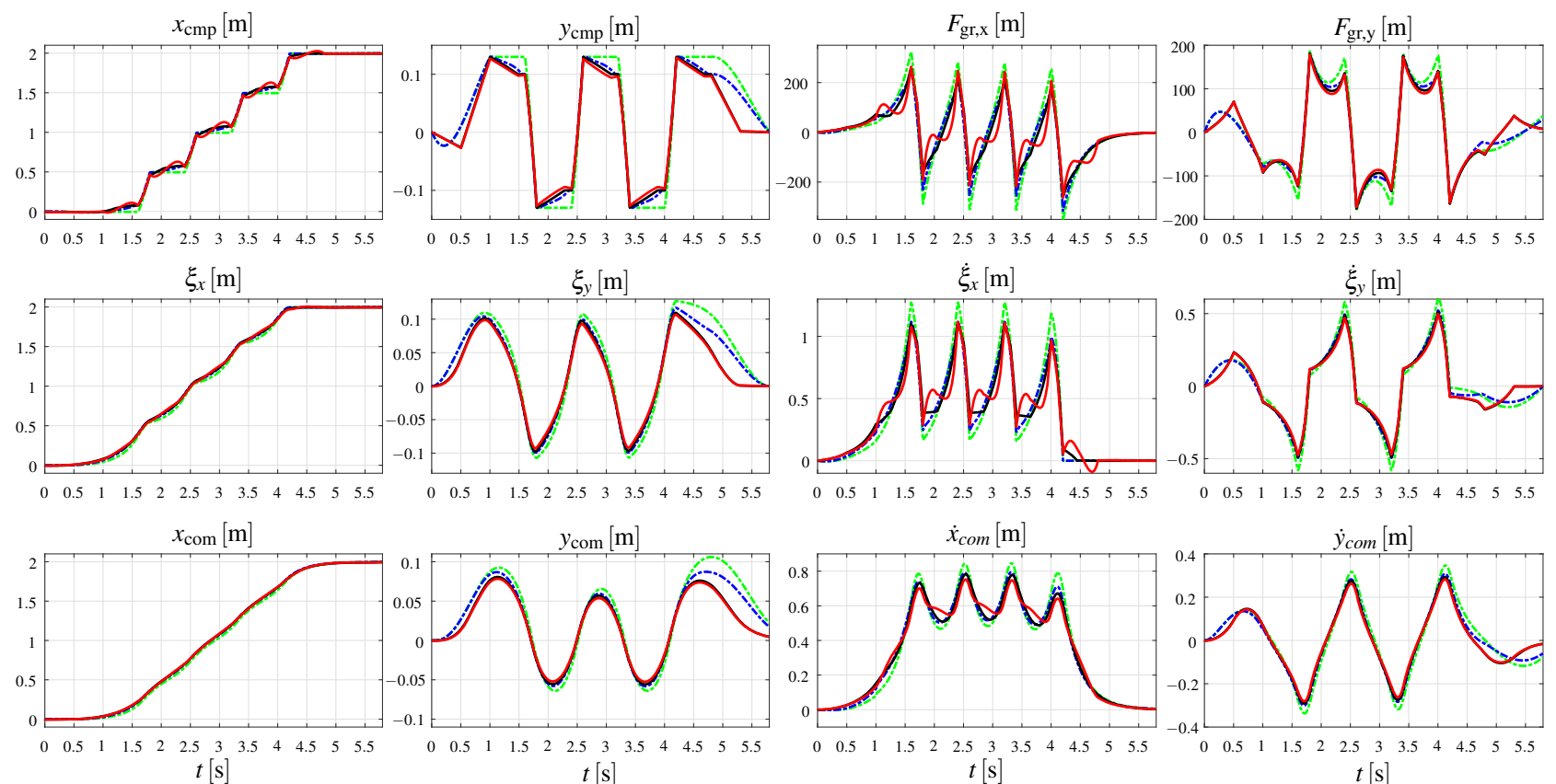

Fig. 4: Comparison of CDS (green dotted), HT (blue dotted), smooth ICP (black) and smooth ICP with angular momentum (red) trajectory generators. An ideal 5-step straight walking plan with parameters $t_{S S}=0.6 \mathrm{~s}, t_{D S}=0.2 \mathrm{~s}, l_{\text {step }}=0.5 \mathrm{~m}$ and $w_{\text {step }}=0.25 \mathrm{~m}$ was used.
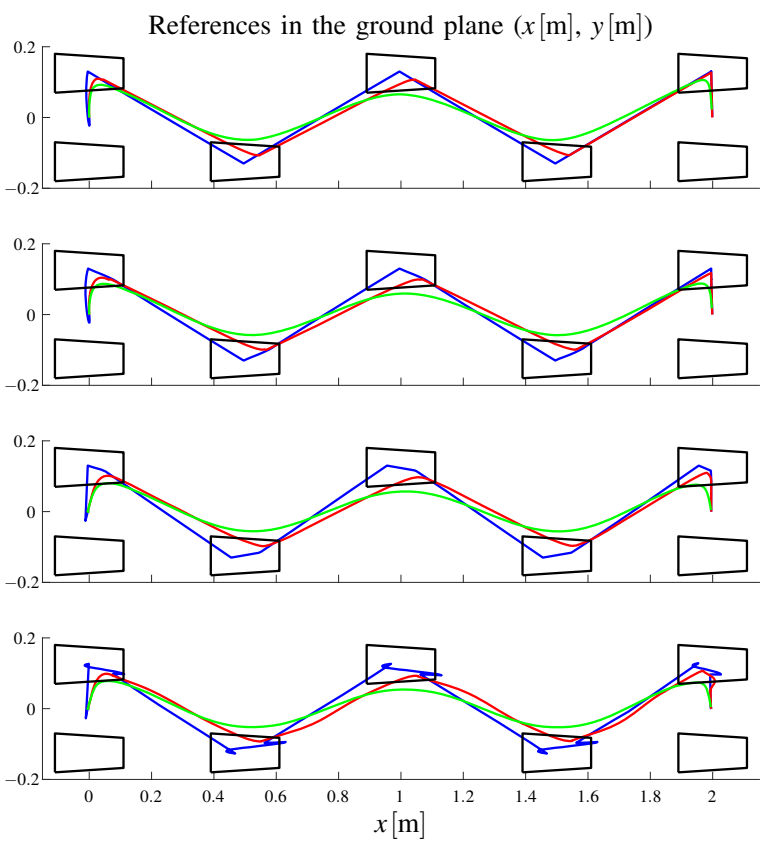

Fig. 5: Top to bottom: CDS, HT, smooth ICP, smooth ICP with angular momentum. The reference CMP (blue), ICP (red) and CoM (green) are shown as projections on the ground plane.
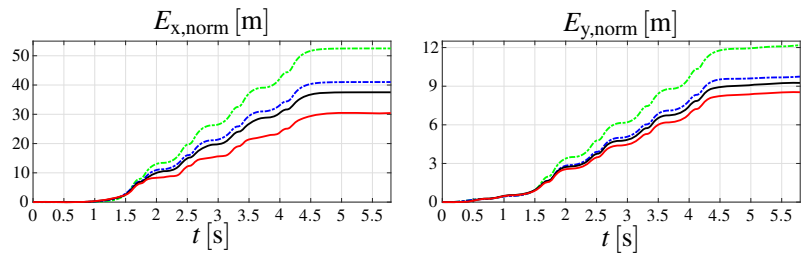

Fig. 6: Comparison of weight normalized energy $\boldsymbol{E}$ for the four planners: CDS (green), HT (blue), smooth ICP (black) and smooth ICP with momentum estimation (red). The latter reduces the cumulative energy required during the gait cycle visibly. is visible that the proposed planner results in a more dynamic motion than the ones ensuing from the other planners. Along the $x$-direction, the estimation-based compensation of swingleg angular momentum decreased ground reaction forces in $x$ during the swing phase considerably and renders them close to zero. It follows that the center of mass acceleration is reduced and less work is done against the CoM inertia. Referring to Fig. 4, both ICP and CoM motion along $x$ is much more linear during gait. While the effect of momentum compensation is less in the frontal plane, a reduction in $y$ sway is still visible. Fig. 5 displays the reference trajectories projected to the ground plane. Comparing the two bottom rows, the effect of accounting for swing leg momentum on the CMP (blue) is clearly visible, while the CoM (green) is shifted less from side to side. Based on the LIP model, the external energy requirements of the resulting gaits can be computed from the ground reaction forces and the CoM velocity as an indicator of gait smoothness [19]. Fig. 6 provides the weight normalized energies. It can be observed that the proposed planner yields a significantly more efficient locomotion pattern than the other planners. This can be ascribed to both the reduced cumulative jerk of the CoM trajectory and the more dynamic gait with less CoM sway.

\section{B. Atlas Simulation}

We tested the proposed planner's performance in simulation on Boston Dynamics' Atlas robot using IHMC's Simulation Construction Set (SCS). The scenarios we evaluated were undisturbed straight walking, force-disturbed straight walking and force-disturbed side stepping. Each step plan was parameterized with the default values used in the previous section for ideal planning: swing time $t_{S S}=0.6 \mathrm{~s}$, transfer time $t_{D S}=0.2 \mathrm{~s}$, step length $l_{\text {step }}=0.5 \mathrm{~m}$ and step 
width $w_{\text {step }}=0.25 \mathrm{~m}$. The results are presented below.

1) Straight Walking: Our plan consisted of 5 forward steps with an initial standing-to-walking transition and a final walking-to-standing transition. The resulting measured ICP, ground reaction forces and weight normalized external energy can be observed in Fig. 7. Similarly to the ideal planning case, the measured ICP exhibits a much more linear movement along $x$ when angular momentum is accounted for, while side to side sway is slightly reduced (top row). Looking at the ground reaction forces, it is visible that force magnitudes are greatly reduced during the swing phase, while on average the instantaneous impact force during transition into double support shows a higher peak. As the peak forces are only encountered for a very short duration and the percentage increase in magnitude is small, we were unable to see a degraded performance of the planner with angular momentum compensation enabled. As our current angular momentum model looses accuracy during the transfer phase, we furthermore believe that these instantaneous peaks could be mitigated by incorporating a more extensive model. Judging by the bottom row, the required external energy is visibly lowered for aforementioned reasons and the proposed planner seems to outperform previous versions.

2) Disturbed Straight Walking: In order to evaluate robustness, we simulated forward walking under force disturbances for all four planners on a 8-step plan. During walking, the robot is first subjected to a force acting along $y$, $F_{y}=-40 \mathrm{~N}$, followed by a force acting along $x, F_{x}=100 \mathrm{~N}$. Both forces are being applied at the robot's chest for a duration of 1s. Both the HT planner and the smooth planner without angular momentum do not withstand the first force and the controller fails to stabilize the system. The CDS planner manages to withstand the first force by not breaking contact with its swing foot throughout the duration of force application, but fails to withstand the second force. Only the smooth planner with momentum compensation remains stable and carries out the entire plan. Fig. 8 provides the measured ICP along $x$ and $y$, as well as the corresponding footholds (red) adjusted from the nominal footholds (grey).

3) Disturbed Side Stepping: In order to gain insights into possible directionality of the planners' performances, we tested side stepping under force disturbances. The plan encompassed 10 steps, while forces were applied along $y$, $F_{y}=-100 \mathrm{~N}$, and $x, F_{x}=70 \mathrm{~N}$ for a duration of $1 \mathrm{~s}$ each. Based on Fig. 9, it can be observed that the CDS planner (green), the HT planner (blue) and the smooth ICP planner (black) failed to complete the task. The impact of the first force pushed the robot into a configuration from which it was unable to recover in all three cases. Only the smooth ICP planner with angular momentum (red) successfully completed the task and therefore seems to offer the greatest robustness under the conditions tested.
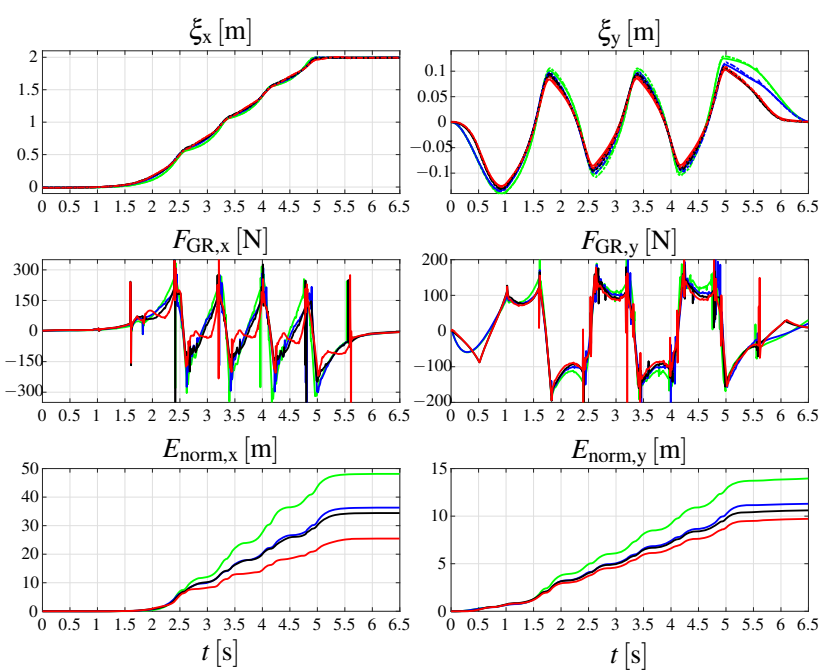

Fig. 7: Top to bottom: comparison of measured ICP $\boldsymbol{\xi}$, ground reaction forces $\boldsymbol{F}_{G R}$ and weight normalized energy $\boldsymbol{E}_{\text {norm }}$. Angular momentum estimation (red) reduces the required sagittal force during swing visibly and in turn reduces the external energy requirements of the gait.
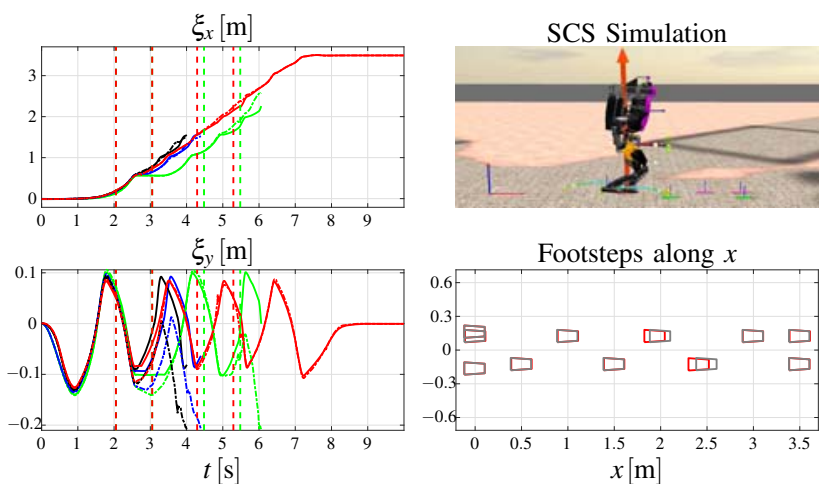

Fig. 8: Measured ICP for a straight walk under force perturbation (duration marked with vertical lines) for the four planners: CDS (green), HT (blue), smooth ICP (black) and smooth ICP with momentum estimation (red). Only the latter was able to complete the task. Its adjusted footsteps are shown in the bottom right.
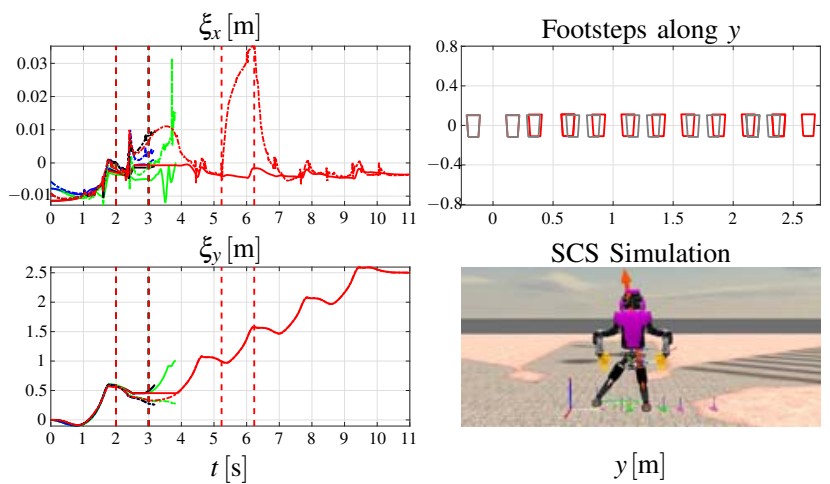

Fig. 9: Measured ICP for side-stepping under force perturbation (duration marked with vertical lines) for the four planners: CDS (green), HT (blue), smooth ICP (black) and smooth ICP with momentum estimation (red). The latter shows the most robust task completion. Its adjusted footsteps are shown in the top right. 


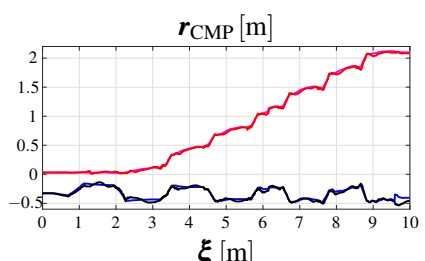

$\boldsymbol{\xi}[\mathrm{m}]$
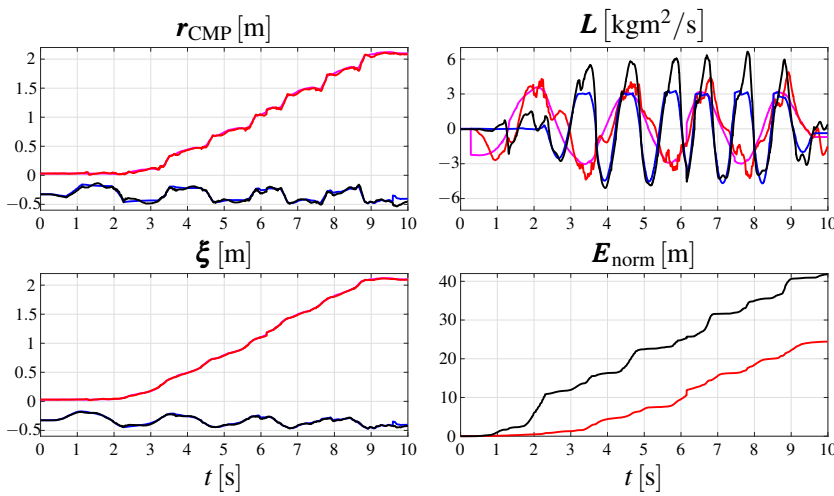

Fig. 10: Experimental results for a straight walk with the Atlas humanoid. Left: CMP (top) and ICP (bottom). Right: angular momentum (top) and normalized external energy (bottom). Desired values are given in magenta and blue, estimated values in red and black for $x$ and $y$, respectively.

\section{EXPERIMENTAL RESULTS}

The proposed planner with momentum compensation was successfully tested on the physical Atlas humanoid robot. Atlas was able to perform flat ground walking, including aggressive turning, sidestepping and backward walking. We were furthermore able to perform step up and step down, as well as traversing both a setup of randomly oriented cinder blocks and a gravel pit (see accompanying video). The new planner therefore met all the benchmark requirements to be run continuously on the real hardware. Below, we show data obtained from executing an 8-step plan on flat ground, parameterized using swing time $t_{S S}=0.9 \mathrm{~s}$, transfer time $t_{D S}=0.35 \mathrm{~s}$, step length $l_{\text {step }}=0.25 \mathrm{~m}$ and step width $w_{\text {step }}=$ $0.25 \mathrm{~m}$. Based on Fig. 10, it can be observed that the angular momentum profile predicted from the very lightweight implementation of Section III-A.2 offers a reasonable approximation to the actual angular momentum. Because our main focus was on the sagittal plane, the prediction is better along $x$ than it is along the $y$ direction. Considering this angular momentum reference in the trajectory generation resulted in the CMP and ICP reference trajectories provided in left column of Fig. 10. The desired values are tracked sufficiently well and little disagreement between them and the recorded data is observed. Furthermore, the estimated external energy required for the step plan is provided in its weight normalized form and seems to be in general agreement with the simulation results.

\section{CONClusion And Future Work}

In this paper, we proposed an extension to the smooth ICP planner proposed in [13] that incorporates predicted centroidal angular momentum trajectories at the planning stage. We were able to reformulate the planner from being linear in the waypoints to being linear in the spline coefficients. This allowed us to write the reference CMP trajectory as a simple addition of reference $\mathrm{CoP}$ and reference angular momentum coefficients. Integrating the resulting formulation twice resulted in a closed form representation for both the reference ICP and reference CoM trajectories.
Furthermore, we provided a very lightweight approximation to the centroidal angular momentum profile during the gait cycle that worked sufficiently well to reduce the external energy requirements of the tested gait. The proposed planner can work on both an online estimation of angular momentum or a pre-planned trajectory taken e.g. from animation. It was thoroughly tested in a simulation of Boston Dynamic's Atlas humanoid robot and successfully deployed on the real hardware. Future work will include the incorporation of a more sophisticated angular momentum approximation scheme capable of capturing higher order dynamic interactions and that offers a more accurate prediction during the transfer phase of gait.

\section{REFERENCES}

[1] S. Kajita, F. Kanehiro, K. Kaneko, K. Yokoi, and H. Hirukawa, "The 3d linear inverted pendulum mode: A simple modeling for a biped walking pattern generation," in IEEE/RSJ International Conference on Intelligent Robots and Systems (IROS), vol. 1, 2001, pp. 239-246.

[2] M. Vukobratovic and J. Stepanenko, "On the stability of anthropomorphic systems," Mathematical Biosciences, vol. 15, no. 1, pp. 1 - 37, 1972.

[3] S. Kajita, F. Kanehiro, K. Kaneko, K. Fujiwara, K. Harada, K. Yokoi, and $\mathrm{H}$. Hirukawa, "Biped walking pattern generation by using preview control of zero-moment point," in IEEE International Conference on Robotics and Automation (ICRA), vol. 2, 2003, pp. 1620-1626.

[4] J. Pratt, J. Carff, S. Drakunov, and A. Goswami, "Capture point: A step toward humanoid push recovery," in IEEE-RAS International Conference on Humanoid Robots (Humanoids), 2006, pp. 200-207.

[5] J. Englsberger, C. Ott, M. A. Roa, A. Albu-Schäffer, and G. Hirzinger, "Bipedal walking control based on capture point dynamics," in IEEE/RSJ International Conference on Intelligent Robots and Systems (IROS), 2011, pp. 4420-4427.

[6] T. Koolen, T. De Boer, J. Rebula, A. Goswami, and J. Pratt, "Capturability-based analysis and control of legged locomotion, part 1 : Theory and application to three simple gait models," The International Journal of Robotics Research, vol. 31, no. 9, pp. 1094-1113, 2012.

[7] J. Englsberger, C. Ott, and A. Albu-Schäffer, "Three-dimensional bipedal walking control using divergent component of motion," in IEEE/RSJ International Conference on Intelligent Robots and Systems (IROS), 2013, pp. 2600-2607.

[8] M. A. Hopkins, D. W. Hong, and A. Leonessa, "Humanoid locomotion on uneven terrain using the time-varying divergent component of motion," in IEEE-RAS International Conference on Humanoid Robots (Humanoids), 2014, pp. 266-272.

[9] H. Herr and M. Popovic, "Angular momentum in human walking," Journal of Experimental Biology, vol. 211, no. 4, pp. 467-481, 2008.

[10] S.-H. Lee and A. Goswami, "Reaction mass pendulum (rmp): An explicit model for centroidal angular momentum of humanoid robots,' in IEEE International Conference on Robotics and Automation (ICRA), 2007, pp. 4667-4672.

[11] H. Dai, A. Valenzuela, and R. Tedrake, "Whole-body motion planning with centroidal dynamics and full kinematics," in IEEE-RAS International Conference on Humanoid Robots (Humanoids), 2014, pp. 295302.

[12] A. Herzog, N. Rotella, S. Schaal, and L. Righetti, "Trajectory generation for multi-contact momentum control," in IEEE-RAS 15th International Conference on Humanoid Robots (Humanoids), 2015, pp. 874-880.

[13] J. Englsberger, G. Mesesan, and C. Ott, "Smooth trajectory generation and push-recovery based on divergent component of motion," in IEEE/RSJ International Conference on Intelligent Robots and Systems (IROS), 2017.

[14] J. Englsberger, "Combining reduced dynamics models and whole-body control for agile humanoid locomotion," Ph.D. dissertation, Technische Universität München, 2016.

[15] T. Koolen, S. Bertrand, G. Thomas, T. de Boer, T. Wu, J. Smith, J. Englsberger, and J. E. Pratt, "Design of a momentum-based control framework and application to the humanoid robot atlas." International Journal of Humanoid Robotics, vol. 13, no. 1, 2016. 
[16] M. B. Popovic, A. Goswami, and H. Herr, "Ground reference points in legged locomotion: Definitions, biological trajectories and control implications," The International Journal of Robotics Research, vol. 24, no. 12, pp. 1013-1032, 2005.

[17] J. Englsberger, T. Koolen, S. Bertrand, J. Pratt, C. Ott, and A. AlbuSchäffer, "Trajectory generation for continuous leg forces during double support and heel-to-toe shift based on divergent component of motion," in IEEE/RSJ International Conference on Intelligent Robots and Systems (IROS), 2014, pp. 4022-4029.

[18] T. Takenaka, T. Matsumoto, and T. Yoshiike, "Real time motion generation and control for biped robot-1 st report: Walking gait pattern generation," in IEEE/RSJ International Conference on Intelligent Robots and Systems (IROS), 2009, pp. 1084-1091.

[19] V. M. Zatsiorsky and R. J. Gregor, "Mechanical power and work in human movement," in Energetics of Human Activity, W. A. Sparrow, Ed. Human Kinetics, 2000, ch. 7, pp. 195-227. 